Please do not remove this page

RMIT

UNIVERSITY

\title{
Consensus of second-order multi-agent systems with delayed nonlinear dynamics and intermittent communications
}

Wen, Guanghui; Duan, Zhisheng; Yu, Wenwu; Chen, Guanrong GR

https://researchrepository.rmit.edu.au/esploro/outputs/9921859251001341/filesAndLinks?institution=61RMIT_INST\&index=null

Wen, G., Duan, Z., Yu, W., \& Chen, G. G. (2013). Consensus of second-order multi-agent systems with delayed nonlinear dynamics and intermittent communications. International Journal of Control, 86(2), 322-331. https://doi.org/10.1080/00207179.2012.727473

Published Version: https://doi.org/10.1080/00207179.2012.727473

Repository homepage: https://researchrepository.rmit.edu.au

(c) 2013 Taylor \& Francis Group, LLC.

Downloaded On 2023/04/26 15:19:53 +1000 
Thank you for downloading this document from the RMIT Research Repository.

The RMIT Research Repository is an open access database showcasing the research outputs of RMIT University researchers.

RMIT Research Repository: http://researchbank.rmit.edu.au/

\section{Citation:}

Wen, G, Duan, Z, Yu, W and Chen, G 2013, 'Consensus of second-order multi-agent systems with delayed nonlinear dynamics and intermittent communications', International Journal of Control, vol. 86, no. 2, pp. 322-331.

See this record in the RMIT Research Repository at:

http://researchbank.rmit.edu.au/view/rmit:20285

Version: Submitted Version

Copyright Statement: (c) 2013 Taylor \& Francis Group, LLC.

Link to Published Version:

http://dx.doi.org/10.1080/00207179.2012.727473 


\title{
Second-order consensus of multi-agent systems with delayed nonlinear dynamics and intermittent measurements
}

\author{
Guanghui Wen ${ }^{\mathrm{a}}$, Zhisheng Duan ${ }^{\mathrm{a}}$, Wenwu $\mathrm{Yu}^{\mathrm{b}, *}$, Guanrong Chen ${ }^{\mathrm{b}}$ \\ ${ }^{a}$ State Key Laboratory for Turbulence and Complex Systems, College of Engineering, Peking University, Beijing 100871, P. R. China \\ ${ }^{b}$ Department of Electronic Engineering, City University of Hong Kong, Hong Kong, SAR, P. R. China
}

\begin{abstract}
This paper investigates second-order consensus of multi-agent systems with delayed nonlinear dynamics and switching topologies. Each agent is assumed to obtain the measurements of relative states between its own and the neighbors only at a sequence of disconnected time intervals. A novel intermittent consensus protocol is proposed to guarantee the states of agents with time-varying velocities to reach second-order consensus under a fixed strongly connected and balanced network topology. The results are then extended to second-order consensus in multi-agent systems with switching topologies, where each possible communication topology is strongly connected and balanced. By virtue of the Lyapunov control approach, it is shown that consensus can be reached if the general algebraic connectivity and communication time duration are larger than their corresponding threshold values respectively. Finally, simulation examples are provided to verify the theoretical analysis and effectiveness of the new protocol.
\end{abstract}

Keywords: Multi-agent system, second-order consensus, intermittent measurement, delayed nonlinear dynamics.

\section{Introduction}

Recently, cooperative control has received considerable attention for its wide applications in multi-agents systems, where typical examples include state-consensus seeking of multiple mobile vehicles [1,2,3], design of distributed sensor networks [4], and control of flocking and rendezvous in natural as well as social systems [5, 6, 7]. Among the numerous research topics in cooperative control, consensus problem received particular interests $[8,9,10]$, which can be generally described as how to design an appropriate protocol based on local information under some communication topology to ensure the multiple agents to reach an agreement on certain quantities of interest.

In Ref. [5], Vicsek et al. introduced an interesting discrete-time model of mobile agents, where each agent's motion is updated according to a local rule based on its own state as well as the states of its neighbors. Some theoretical analysis of the consensus problem on the linearized Vicsek's mode was provided in Ref. [8]. Then, in Ref. [9], a general framework of the consensus problem for networks of dynamic agents with a fixed or switching topology and communication time-delays was established. The consensus conditions derived in Ref. [9] were further relaxed in Ref. [10]. In addition, consensus over a random communication topology [11, 12], asynchronous consensus [13, 14, 15], high-dimensional consensus [16], consensus problems with nonlinear protocols [17, 18] and communication noises $[19,20]$, have been investigated. Note that most of the above-mentioned works are concerned with the first-order

\footnotetext{
${ }^{*}$ Corresponding author.

Email addresses: wenguanghui@gmail.com (Guanghui Wen), duanzs@pku.edu.cn (Zhisheng Duan), wenwuyu@gmail.com (Wenwu $\mathrm{Yu}$ ), eegchen@cityu. edu.hk (Guanrong Chen)
} 
consensus problem, where each agent is governed by first-order dynamics. In reality, however, a large class of multiagent systems are modeled by second-order dynamics [21], [22], [23] [24], [25], [26]. In Ref. [21], the second-order consensus problem with zero initial and finial consensus velocities under undirected communication topology was investigated. Taking into account the general case where information flows may be unidirectional due to sensors with limited sensing ranges or multi-agents with directed communication links, a new kind of second-order consensus problems under directed communication topologies was discussed in Refs. [22, 23]. Some sufficient conditions were obtained for achieving second-order consensus, and it was shown that the communication topology having a spanning tree is not a sufficient condition for reaching second-order consensus, which is different in kind from the first-order consensus problems [22, 23, 24]. Then, in Refs. [27, 28, 29], some necessary and sufficient conditions for second-order consensus in directed networks were derived. Concerning that transmission time-delay is a key factor influencing the stability of consensus in linear multi-agent systems, a necessary and sufficient condition was obtained in Ref. [29] for second-order consensus in networked multi-agent systems with transmission delays. In contrast to the aforementioned second-order consensus algorithms [21, 22, 24, 27, 28], where the consensus velocity is a constant, a consensus algorithm in coupled second-order linear harmonic oscillators with asymptotic periodic velocity and directed communication topology was considered in Ref. [30]. The dynamical model studied in Ref. [30] in essence is a second-order multi-agent system with linear intrinsic dynamics. A more general case is that each agent has nonlinear dynamics $[31,32,33,34,35,36]$. From this perspective, Yu et al. investigated the second-order consensus problem in multi-agent systems with nonlinear dynamics and directed topologies in Ref. [37], where by using tools from the algebraic graph theory and Lyapunov control approach, some sufficient conditions were derived for reaching second-order consensus with time-varying consensus velocities.

It should be noticed that most of the aforementioned works on second-order consensus problems in multi-agent systems, it was assumed that information is transmitted continuously among multi-agents. However, this may not be the case in reality due to technological limitations or external disturbances. For example, in some cases, agents can only obtain the measurements of states of its neighbors intermittently due to the limited sensing abilities. To deal with this challenging situation, a novel intermittent consensus protocol is proposed in this paper to guarantee second-order consensus. On the other hand, in order to analyze the second-order consensus problem in multi-agent systems within a general framework, delayed nonlinear dynamics are introduced into the model of each agent in this paper. By virtue of the Lyapunov control approach, some sufficient conditions are derived for reaching second-order consensus with time-varying agent velocities.

The rest of the paper is organized as follows. In Section 2, some preliminaries in algebraic graph theory and the model formulation are given. In Section 3, second-order consensus problems with delayed nonlinear dynamics and intermittent measurements under fixed and switching strongly connected and balanced communication topologies are investigated, respectively. In Section 4, simulation examples are provided to verify the theoretical results. Conclusions are finally drawn in Section 5.

The following notations are used throughout the paper. Let $\mathbb{R}$ and $\mathbb{N}$ be the sets of real and natural numbers, respectively. $\mathbb{R}^{N}$ is the $\mathrm{N}$-dimensional real vector space and $\|\cdot\|$ denotes the Euclidian norm. $\mathbb{R}^{N \times N}$ is $N \times N$ real matrix space. Let $I_{N}\left(O_{N}\right)$ be the $N$-dimensional identity (zero) matrix, and $1_{N}\left(0_{N}\right)$ be the $N$-dimensional column vector with each entry being $1(0)$. Suppose that matrix $M \in \mathbb{R}^{N \times N}$ has real eigenvalues, with $\lambda_{i}(M)$ being the $i$ th smallest eigenvalue $(1 \leq i \leq N)$. Notation $\otimes$ represents the Kronecker product. Furthermore, a column vector $x \in \mathbb{R}^{N}$ is said to be positive if every entry $x_{i}>0(1 \leq i \leq N)$. 


\section{Preliminaries}

In this section, some preliminaries in algebraic graph theory and model formulation for second-order consensus in multi-agent systems with delayed nonlinear dynamics and intermittent measurements are introduced.

\subsection{Algebraic graph theory}

Let $\mathcal{G}(\mathcal{V}, \mathcal{E}, \mathcal{A})$ be a directed graph with the set of nodes $\mathcal{V}=\left\{v_{1}, v_{2}, \cdots, v_{N}\right\}$, the set of directed edges $\mathcal{E} \subseteq \mathcal{V} \times \mathcal{V}$, and a weighted adjacency matrix $\mathcal{A}=\left[a_{i j}\right]_{N \times N}$ with non-negative adjacency elements $a_{i j}$. An edge $e_{i j}$ in graph $\mathcal{G}$ is denoted by the ordered pair of nodes $\left(v_{j}, v_{i}\right)$, where $v_{j}$ and $v_{i}$ are called the parent and child nodes, respectively, and $e_{i j} \in \mathcal{E}$ if and only if $a_{i j}>0$. Furthermore, self-loops are not allowed, i.e., $a_{i i}=0$ for all $i=1,2, \cdots N$. For simplicity, denote $\mathcal{G}(\mathcal{V}, \mathcal{E}, \mathcal{A})$ by $\mathcal{G}(\mathcal{A})$ if no confusion will arise.

A directed path from node $v_{i}$ to $v_{j}$ is a finite ordered sequence of edges, $\left(v_{i}, v_{k_{1}}\right),\left(v_{k_{1}}, v_{k_{2}}\right), \cdots,\left(v_{k_{l}}, v_{j}\right)$, with distinct nodes $v_{k_{m}}, m=1,2, \cdots, l$. A directed graph is called strongly connected if and only if there is a directed path between any pair of distinct nodes. Moreover, a directed graph $\mathcal{G}(\mathcal{A})$ is called balanced if

$$
\sum_{j} a_{i j}=\sum_{j} a_{j i}, \quad \forall i=1,2, \cdots, N .
$$

The Laplacian matrix $L=\left[l_{i j}\right]_{N \times N}$ of $\mathcal{G}(\mathcal{A})$ is defined as

$$
l_{i j}= \begin{cases}-a_{i j}, & i \neq j \\ \sum_{k=1, k \neq i}^{N} a_{i k}, & i=j\end{cases}
$$

For a directed graph, the Laplacian matrix $L$ has the following properties.

Lemma 1: ([10]) Suppose that a directed graph $\mathcal{G}(\mathcal{A})$ is strongly connected. Then, 0 is a simple eigenvalue of its Laplacian matrix $L$, and all the other eigenvalues of $L$ have positive real parts.

Lemma 2: ([9]) A directed graph $\mathcal{G}(\mathcal{A})$ is balanced if and only if $1_{N}$ is the left eigenvector of its Laplacian matrix $L$ associated with zero eigenvalue, i.e. $1_{N}^{T} L=0$.

For an undirected graph, its Laplacian matrix $L$ is positive semi-definite. For a connected undirected graph, there is one simple zero eigenvalue of $L$, and all the other eigenvalues of $L$ are positive and real.

\subsection{Formulation of the model}

Consider a group of $N$ agents indexed by $1,2, \cdots, N$. The commonly studied continuous-time second-order protocol of the $N$ agents is described as follows [23, 24, 26]:

$$
\left\{\begin{array}{l}
\dot{x}_{i}(t)=v_{i}(t) \\
\dot{v}_{i}(t)=-\alpha \sum_{j=1}^{N} l_{i j} x_{j}(t)-\beta \sum_{j=1}^{N} l_{i j} v_{j}(t), \quad i=1,2, \cdots, N,
\end{array}\right.
$$

where $x_{i} \in \mathbb{R}^{n}$ and $v_{i} \in \mathbb{R}^{n}$ are the position and velocity states of the $i$ th agent, respectively, $\alpha$ and $\beta$ represent the coupling strengths, $L=\left[l_{i j}\right]_{N \times N}$ is the Laplacian matrix of the fixed communication topology $\mathcal{G}(\mathcal{A})$. When the agents reach second-order consensus, the velocities of all agents converge to $\sum_{j=1}^{N} \xi_{j} v_{j}(0)$, which depends only on the initial velocities of the agents, where $\xi=\left(\xi_{1}, \cdots, \xi_{N}\right)$ is the nonnegative left eigenvector of $L$ associated with the eigenvalue 0 satisfying $\xi^{T} 1_{N}=1[23,24]$. However, in most applications of multi-agent formations, the velocity of each agent 
is generally evolving nonlinearly. Therefore, Yu et al. proposed the following second-order consensus protocol with nonlinear dynamics [37]

$$
\left\{\begin{array}{l}
\dot{x}_{i}(t)=v_{i}(t) \\
\dot{v}_{i}(t)=f\left(x_{i}(t), v_{i}(t), t\right)-\alpha \sum_{j=1}^{N} l_{i j} x_{j}(t)-\beta \sum_{j=1}^{N} l_{i j} v_{j}(t), \quad i=1,2, \cdots, N,
\end{array}\right.
$$

where $f: \mathbb{R}^{n} \times \mathbb{R}^{n} \times \mathbb{R}^{+} \rightarrow \mathbb{R}^{n}$ is a continuously differentiable vector-valued function. In some cases, $f$ can be taken as $f=-\nabla U(x, v)$, where $U(x, v)$ is a potential function, thus the multi-agent system (4) includes many popular swarming and flocking models [38], [39] as special cases.

Note that most of the existing protocols are implemented based on a common assumption that all information is transmitted continuously among agents. However, in some real situations, agents may only communicate with their neighbors over some disconnected time intervals due to the unreliability of communication channels, failure of physical devices, and limitations of sensing ranges, etc. Motivated by this observation and based on the above-mentioned works $[23,24,37]$, in this paper the following consensus protocol with time-delay and intermittent measurements is considered:

$$
\left\{\begin{array}{l}
\dot{x}_{i}(t)=v_{i}(t) \\
\dot{v}_{i}(t)=f\left(x_{i}(t-\tau), v_{i}(t-\tau), x_{i}(t), v_{i}(t), t\right)-\alpha \sum_{j=1}^{N} l_{i j} x_{j}(t)-\beta \sum_{j=1}^{N} l_{i j} v_{j}(t), \quad t \in[k \omega, k \omega+\delta], \\
\dot{v}_{i}(t)=f\left(x_{i}(t-\tau), v_{i}(t-\tau), x_{i}(t), v_{i}(t), t\right), \quad t \in(k \omega+\delta,(k+1) \omega), \quad k \in \mathbb{N}, \quad i=1,2, \cdots, N
\end{array}\right.
$$

where $f: \mathbb{R}^{n} \times \mathbb{R}^{n} \times \mathbb{R}^{n} \times \mathbb{R}^{n} \times \mathbb{R}^{+} \rightarrow \mathbb{R}^{n}$ is a continuously differentiable vector-valued function representing the intrinsic delayed nonlinear dynamics of agent $i, \tau>0$ is the time-delay constant, and the communication time duration $\delta$ satisfies $\tau<\delta \leq \omega$. Furthermore, $x_{i}(t)=\phi_{i}(t), v_{i}(t)=\psi_{i}(t)$, for $t \in[-\tau, 0], i=1,2, \cdots$, and the initial functions $\phi_{i}$ and $\psi_{i}$ are continuous for $t \in[-\tau, 0]$.

Clearly, since $\sum_{j=1}^{N} l_{i j}=0$, if consensus can be achieved, it is natural to require a solution $s(t)=\left(s_{1}^{T}(t), s_{2}^{T}(t)\right)^{T} \in$ $\mathbb{R}^{2 n}$ of the system (5) be a possible trajectory of an isolated node satisfying

$$
\left\{\begin{array}{l}
\dot{s}_{1}(t)=s_{2}(t) \\
\dot{s}_{2}(t)=f\left(s_{1}(t-\tau), s_{2}(t-\tau), s_{1}(t), s_{2}(t), t\right) .
\end{array}\right.
$$

Here, $s(t)$ may be an isolated equilibrium point, a periodic orbit, or even a chaotic orbit in applications.

Remark 1: If $\tau=0$ and $\delta=\omega$ in system (5), that is, each agent can communicate with its neighbors all the time and the node dynamics depend only on its current states, then system (5) becomes the system (3) studied in Ref. [37].

Lemma 3: (Schur complement [40]) The following linear matrix inequality (LMI),

$$
S=\left(\begin{array}{ll}
S_{11} & S_{12} \\
S_{21} & S_{22}
\end{array}\right)>0,
$$

where $S_{11}=S_{11}^{T}, S_{12}=S_{21}^{T}, S_{22}=S_{22}^{T}$, is equivalent to one of the following conditions:

(i) $S_{11}>0, S_{22}-S_{21} S_{11}^{-1} S_{12}>0$;

(ii) $S_{22}>0, S_{11}-S_{12} S_{22}^{-1} S_{21}>0$.

Lemma 4: (Halanay Inequality [41]) Suppose that the non-negative function $y(t), t \in[-\tau,+\infty$ ), satisfies

$$
\frac{d y(t)}{d t} \leq-c_{1} y(t)+c_{2} y(t-\tau), \quad t \geq 0
$$


where constants $c_{1}>c_{2}>0$. Then,

$$
y(t) \leq|y(0)|_{\tau} e^{-r t}, \quad t \geq 0,
$$

where $|y(0)|_{\tau}=\max _{-\tau \leq s \leq 0} y(s)$ and $r$ is the unique solution of

$$
-r=-c_{1}+c_{2} e^{r \tau}
$$

Lemma 5: [42] Suppose that the non-negative function $y(t), t \in[-\tau, \infty)$, satisfies

$$
\frac{d y(t)}{d t} \leq c_{1} y(t)+c_{2} y(t-\tau), \quad t \geq 0
$$

where $c_{1}, c_{2}$ are positive constants. Then,

$$
y(t) \leq|y(0)|_{\tau} e^{\left(c_{1}+c_{2}\right) t}, \quad t \geq 0
$$

where $|y(0)|_{\tau}=\max _{-\tau \leq s \leq 0} y(s)$

\section{Main Results}

In this section, second-order consensus problems in strongly connected and balanced networks with time-delayed nonlinear dynamics and intermittent measurements are investigated.

Assumption 1: There exist nonnegative constants $\rho_{i}, i \in\{1,2,3,4\}$, such that

$$
\left\|f\left(x_{1}, x_{2}, x_{3}, x_{4}, t\right)-f\left(y_{1}, y_{2}, y_{3}, y_{4}, t\right)\right\| \leq \sum_{i=1}^{4} \rho_{i}\left\|x_{i}-y_{i}\right\|,
$$

$\forall x_{i}, y_{i} \in \mathbb{R}^{n}, i \in\{1,2,3,4\}, t \geq 0$.

Let $\tilde{x}_{i}(t)=x_{i}(t)-\frac{1}{N} \sum_{j=1}^{N} x_{j}(t)$ and $\tilde{v}_{i}(t)=v_{i}(t)-\frac{1}{N} \sum_{j=1}^{N} v_{j}(t)$. One has the following error dynamical system:

$$
\left\{\begin{aligned}
& \dot{\tilde{x}}_{i}(t)= \tilde{v}_{i}(t) \\
& \dot{\tilde{v}}_{i}(t)= f\left(x_{i}(t-\tau), v_{i}(t-\tau), x_{i}(t), v_{i}(t), t\right)-\frac{1}{N} \sum_{j=1}^{N} f\left(x_{j}(t-\tau), v_{j}(t-\tau), x_{j}(t), v_{j}(t), t\right) \\
&-\alpha \sum_{j=1}^{N} l_{i j} \tilde{x}_{j}(t)-\beta \sum_{j=1}^{N} l_{i j} \tilde{v}_{j}(t), \quad k \omega \leq t \leq k \omega+\delta \\
& \dot{\tilde{v}}_{i}(t)= f\left(x_{i}(t-\tau), v_{i}(t-\tau), x_{i}(t), v_{i}(t), t\right)-\frac{1}{N} \sum_{j=1}^{N} f\left(x_{j}(t-\tau), v_{j}(t-\tau), x_{j}(t), v_{j}(t), t\right) \\
& k \omega+\delta<t<(k+1) \omega, \quad i=1, \cdots, N, \quad k=0,1, \cdots
\end{aligned}\right.
$$

Let $\tilde{x}(t)=\left(\tilde{x}_{1}^{T}(t), \cdots, \tilde{x}_{N}^{T}(t)\right)^{T}, \tilde{v}(t)=\left(\tilde{v}_{1}^{T}(t), \cdots, \tilde{v}_{N}^{T}(t)\right)^{T}, f(x(t-\tau), v(t-\tau), x(t), v(t), t)=\left(f^{T}\left(x_{1}(t-\tau), v_{1}(t-\right.\right.$ $\left.\left.\tau), x_{1}(t), v_{1}(t), t\right), \cdots, f^{T}\left(x_{N}(t-\tau), v_{N}(t-\tau), x_{N}(t), v_{N}(t), t\right)\right)^{T}$ and $\tilde{y}(t)=\left(\tilde{x}^{T}(t), \tilde{v}^{T}(t)\right)^{T}$. Then, system (7) can be written as

$$
\begin{cases}\dot{\tilde{y}}(t)=F(x(t-\tau), v(t-\tau), x(t), v(t), t)+\left(B_{1} \otimes I_{n}\right) \tilde{y}(t), & t \in[k \omega, k \omega+\delta], \\ \dot{\tilde{y}}(t)=F(x(t-\tau), v(t-\tau), x(t), v(t), t)+\left(B_{2} \otimes I_{n}\right) \tilde{y}(t), & t \in(k \omega+\delta,(k+1) \omega),\end{cases}
$$


where $F(x(t-\tau), v(t-\tau), x(t), v(t), t)=\left(\begin{array}{c}0_{N n} \\ {\left[\left(I_{N}-\frac{1}{N} 1_{N \times N}\right) \otimes I_{n}\right] f(x(t-\tau), v(t-\tau), x(t), v(t), t)}\end{array}\right), B_{1}=\left(\begin{array}{cc}O_{N} & I_{N} \\ -\alpha L & -\beta L\end{array}\right)$, $B_{2}=\left(\begin{array}{cc}O_{N} & I_{N} \\ O_{N} & O_{N}\end{array}\right)$.

Theorem 1. Suppose that the communication topology $\mathcal{G}(\mathcal{A})$ is strongly connected and balanced, and Assumption 1 holds. Then, second-order consensus in system (5) is achieved if the following conditions hold:

(i) $\lambda_{2}\left(L+L^{T}\right)>\frac{\alpha}{\beta^{2}}$,

(ii) $\lambda_{1}\left(R_{1}\right)>\frac{c_{0} \lambda_{2}\left(P_{1}\right)}{\lambda_{1}(Q)}$,

(iii) $\delta>\frac{r \tau+\left(\gamma_{3}+\gamma_{4}\right) \omega}{r+\gamma_{3}+\gamma_{4}}$,

where $R_{1}=\left(\begin{array}{cc}\left(\alpha \lambda_{2}\left(L+L^{T}\right)-\rho_{1}-\rho_{2}-2 \rho_{3}\right) \alpha & -\left(\beta \rho_{3}+\alpha \rho_{4}\right) \\ -\left(\beta \rho_{3}+\alpha \rho_{4}\right) & \beta^{2} \lambda_{2}\left(L+L^{T}\right)-\left(\rho_{1}+\rho_{2}+2 \rho_{4}\right) \beta-2 \alpha\end{array}\right), P_{1}=\left(\begin{array}{cc}\alpha \beta \lambda_{N}\left(L+L^{T}\right) & \alpha \\ \alpha & \beta\end{array}\right)$, $Q=\left(\begin{array}{cc}\alpha \beta \lambda_{2}\left(L+L^{T}\right) & \alpha \\ \alpha & \beta\end{array}\right), c_{0}=(\alpha+\beta) \max \left\{\rho_{1}, \rho_{2}\right\}, r$ is the unique positive solution of $-r=-\gamma_{1}+\gamma_{2} e^{r \tau}, \quad \gamma_{1}=$ $\frac{\lambda_{1}\left(R_{1}\right)}{\lambda_{2}\left(P_{1}\right)}, \gamma_{2}=\frac{c_{0}}{\lambda_{1}(Q)}, \gamma_{3}=\frac{c_{1}+c_{2}+\sqrt{\left(c_{1}-c_{2}\right)^{2}+c_{3}}}{\lambda_{1}(Q)}, \quad \gamma_{4}=\frac{(\alpha+\beta) \rho_{0}}{\lambda_{1}(Q)}, c_{1}=\frac{\left(\rho_{1}+\rho_{2}+2 \rho_{3}\right) \alpha}{2}, c_{2}=\frac{\left(\rho_{1}+\rho_{2}+2 \rho_{4}\right) \beta}{2}$, and $c_{3}=\left(\beta \rho_{3}+\alpha \rho_{4}+1\right)^{2}$.

Proof: Construct the following Lyapunov function candidate

$$
V(t)=\frac{1}{2} \tilde{y}^{T}(t)\left(P \otimes I_{n}\right) \tilde{y}(t),
$$

where $P=\left(\begin{array}{cc}\alpha \beta\left(L+L^{T}\right) & \alpha I_{N} \\ \alpha I_{N} & \beta I_{N}\end{array}\right)$. It will be shown that $V(t)$ is a valid Lyapunov function for analyzing the error dynamics described by system (8). According to the Courant-Fischer theorem [43], one has

$$
\begin{aligned}
V(t) & =\frac{\alpha \beta}{2} \tilde{x}^{T}(t)\left(\left(L+L^{T}\right) \otimes I_{n}\right) \tilde{x}(t)+\alpha \tilde{x}^{T}(t) \tilde{v}(t)+\frac{\beta}{2} \tilde{v}^{T}(t) \tilde{v}(t) \\
& \geq \frac{1}{2} \tilde{y}^{T}(t)\left(Q \otimes I_{N n}\right) \tilde{y}(t),
\end{aligned}
$$

where $Q=\left(\begin{array}{cc}\alpha \beta \lambda_{2}\left(L+L^{T}\right) & \alpha \\ \alpha & \beta\end{array}\right)$. By Lemma $3, Q>0$ is equivalent to both $\beta>0$ and $\lambda_{2}\left(L+L^{T}\right)>\frac{\alpha}{\beta^{2}}$. From condition (i), one obtains $Q>0, V(t) \geq 0$ and $V(t)=0$ if and only if $\tilde{y}(t)=0_{2 N n}$.

Let $\bar{x}(t-\tau)=\frac{1}{N} \sum_{j=1}^{N} x_{j}(t-\tau), \bar{v}(t-\tau)=\frac{1}{N} \sum_{j=1}^{N} v_{j}(t-\tau), \bar{x}(t)=\frac{1}{N} \sum_{j=1}^{N} x_{j}(t)$, and $\bar{v}(t)=\frac{1}{N} \sum_{j=1}^{N} v_{j}(t)$. For $t \in[k \omega, k \omega+\delta], k \in \mathbb{N}$, taking the time derivative of $V(t)$ along the trajectories of (8) gives

$$
\begin{aligned}
\dot{V}(t)= & \tilde{y}^{T}(t)\left(P \otimes I_{n}\right)\left[F(x(t-\tau), v(t-\tau), x(t), v(t), t)+\left(B_{1} \otimes I_{n}\right) \tilde{y}(t)\right] \\
= & \alpha \tilde{x}^{T}\left[\left(I_{N}-\frac{1}{N} 1_{N \times N}\right) \otimes I_{n}\right] f(x(t-\tau), v(t-\tau), x(t), v(t), t)+\beta \tilde{v}^{T}\left[\left(I_{N}-\frac{1}{N} 1_{N \times N}\right) \otimes I_{n}\right] f(x(t-\tau), v(t-\tau), x(t), v(t), t) \\
& +\frac{1}{2} \tilde{y}^{T}(t)\left[\left(P B_{1}+B_{1}^{T} P\right) \otimes I_{n}\right] \tilde{y}(t) \\
= & {\left[\alpha \tilde{x}^{T}(t)+\beta \tilde{v}^{T}(t)\right]\left[f(x(t-\tau), v(t-\tau), x(t), v(t), t)-1_{N} \otimes f(\bar{x}(t-\tau), \bar{v}(t-\tau), \bar{x}(t), \bar{v}(t), t)\right] } \\
& -\left[\alpha \tilde{x}^{T}(t)+\beta \tilde{v}^{T}(t)\right]\left(\left(\frac{1}{N} 1_{N \times N}\right) \otimes I_{n}\right) f(x(t-\tau), v(t-\tau), x(t), v(t), t) \\
& +\left[\alpha \tilde{x}^{T}(t)+\beta \tilde{v}^{T}(t)\right]\left[1_{N} \otimes f(\bar{x}(t-\tau), \bar{v}(t-\tau), \bar{x}(t), \bar{v}(t), t)\right] \\
& +\frac{1}{2} \tilde{y}^{T}(t)\left[\left(\begin{array}{cc}
-\alpha^{2}\left(L+L^{T}\right) \\
O_{N} \quad-\beta^{2}\left(L+L^{T}\right)+2 \alpha I_{N}
\end{array}\right) \otimes I_{n}\right] \tilde{y}(t)
\end{aligned}
$$


Since $\tilde{x}(t)=\left[\left(I_{N}-\frac{1}{N} 1_{N \times N}\right) \otimes I_{n}\right] x(t)$ and $\tilde{v}(t)=\left[\left(I_{N}-\frac{1}{N} 1_{N \times N}\right) \otimes I_{n}\right] v(t)$, one gets

$$
\begin{aligned}
& \tilde{x}^{T}(t)\left[1_{N} \otimes f(\bar{x}(t-\tau), \bar{v}(t-\tau), \bar{x}(t), \bar{v}(t), t)\right]=0, \\
& \tilde{v}^{T}(t)\left[1_{N} \otimes f(\bar{x}(t-\tau), \bar{v}(t-\tau), \bar{x}(t), \bar{v}(t), t)\right]=0,
\end{aligned}
$$

and

$$
\begin{aligned}
& \tilde{x}^{T}(t)\left[\left(\frac{1}{N} 1_{N \times N}\right) \otimes I_{n}\right] f(x(t-\tau), v(t-\tau), x(t), v(t), t)=0, \\
& \tilde{v}^{T}(t)\left[\left(\frac{1}{N} 1_{N \times N}\right) \otimes I_{n}\right] f(x(t-\tau), v(t-\tau), x(t), v(t), t)=0 .
\end{aligned}
$$

Combining (10)-(12), one obtains

$$
\begin{gathered}
\dot{V}(t)=\left[\alpha \tilde{x}^{T}(t)+\beta \tilde{v}^{T}(t)\right]\left[f(x(t-\tau), v(t-\tau), x(t), v(t), t)-1_{N} \otimes f(\bar{x}(t-\tau), \bar{v}(t-\tau), \bar{x}(t), \bar{v}(t), t)\right] \\
+\tilde{y}^{T}(t)\left[\left(\begin{array}{cc}
-\frac{\alpha^{2}}{2}\left(L+L^{T}\right) & O_{N} \\
O_{N} & -\frac{\beta^{2}}{2}\left(L+L^{T}\right)+\alpha I_{N}
\end{array}\right) \otimes I_{n}\right] \tilde{y}(t) .
\end{gathered}
$$

By Assumption 1, one gets

$$
\begin{aligned}
& \alpha \tilde{x}^{T}(t)\left[f(x(t-\tau), v(t-\tau), x(t), v(t), t)-1_{N} \otimes f(\bar{x}(t-\tau), \bar{v}(t-\tau), \bar{x}(t), \bar{v}(t), t)\right] \\
= & \alpha \sum_{i=1}^{N}\left(x_{i}(t)-\bar{x}(t)\right)^{T}\left[f\left(x_{i}(t-\tau), v_{i}(t-\tau), x_{i}(t), v_{i}(t), t\right)-f(\bar{x}(t-\tau), \bar{v}(t-\tau), \bar{x}(t), \bar{v}(t), t)\right] \\
\leq & \alpha \sum_{i=1}^{N}\left\|\tilde{x}_{i}(t)\right\|\left(\rho_{1}\left\|\tilde{x}_{i}(t-\tau)\right\|+\rho_{2}\left\|\tilde{v}_{i}(t-\tau)\right\|+\rho_{3}\left\|\tilde{x}_{i}(t)\right\|+\rho_{4}\left\|\tilde{v}_{i}(t)\right\|\right) \\
\leq & \left(\frac{\rho_{1}+\rho_{2}}{2}+\rho_{3}\right) \sum_{i=1}^{N}\left\|\tilde{x}_{i}(t)\right\|^{2}+\frac{\rho_{1}}{2} \sum_{i=1}^{N}\left\|\tilde{x}_{i}(t-\tau)\right\|^{2}+\frac{\rho_{2}}{2} \sum_{i=1}^{N}\left\|\tilde{v}_{i}(t-\tau)\right\|^{2}+\rho_{4} \sum_{i=1}^{N}\left\|\tilde{x}_{i}(t)\right\|\left\|\tilde{v}_{i}(t)\right\|,
\end{aligned}
$$

and

$$
\begin{aligned}
& \beta \tilde{v}^{T}(t)\left[f(x(t-\tau), v(t-\tau), x(t), v(t), t)-1_{N} \otimes f(\bar{x}(t-\tau), \bar{v}(t-\tau), \bar{x}(t), \bar{v}(t), t)\right] \\
= & \beta \sum_{i=1}^{N}\left(v_{i}(t)-\bar{v}(t)\right)^{T}\left[f\left(x_{i}(t-\tau), v_{i}(t-\tau), x_{i}(t), v_{i}(t), t\right)-f(\bar{x}(t-\tau), \bar{v}(t-\tau), \bar{x}(t), \bar{v}(t), t)\right] \\
\leq & \beta \sum_{i=1}^{N}\left\|\tilde{v}_{i}(t)\right\|\left(\rho_{1}\left\|\tilde{x}_{i}(t-\tau)\right\|+\rho_{2}\left\|\tilde{v}_{i}(t-\tau)\right\|+\rho_{3}\left\|\tilde{x}_{i}(t)\right\|+\rho_{4}\left\|\tilde{v}_{i}(t)\right\|\right) \\
\leq & \beta\left(\frac{\rho_{1}}{2} \sum_{i=1}^{N}\left\|\tilde{x}_{i}(t-\tau)\right\|^{2}+\left(\frac{\rho_{1}+\rho_{2}}{2}+\rho_{4}\right) \sum_{i=1}^{N}\left\|\tilde{v}_{i}(t)\right\|^{2}+\frac{\rho_{2}}{2} \sum_{i=1}^{N}\left\|\tilde{v}_{i}(t-\tau)\right\|^{2}+\rho_{3} \sum_{i=1}^{N}\left\|\tilde{x}_{i}(t)\right\|\left\|\tilde{v}_{i}(t)\right\|\right) .
\end{aligned}
$$


Combining (13)-(15) gives

$$
\begin{aligned}
\dot{V}(t) & \leq \frac{\left(\rho_{1}+\rho_{2}+2 \rho_{3}\right) \alpha}{2} \sum_{i=1}^{N}\left\|\tilde{x}_{i}(t)\right\|^{2}+\frac{(\alpha+\beta) \rho_{1}}{2} \sum_{i=1}^{N}\left\|\tilde{x}_{i}(t-\tau)\right\|^{2}+\frac{\left(\rho_{1}+\rho_{2}+2 \rho_{4}\right) \beta}{2} \sum_{i=1}^{N}\left\|\tilde{v}_{i}(t)\right\|^{2} \\
& +\frac{(\alpha+\beta) \rho_{2}}{2} \sum_{i=1}^{N}\left\|\tilde{v}_{i}(t-\tau)\right\|^{2}+\left(\beta \rho_{3}+\alpha \rho_{4}\right) \sum_{i=1}^{N}\left\|\tilde{x}_{i}(t)\right\|\left\|\tilde{v}_{i}(t)\right\| \\
& +\tilde{y}^{T}(t)\left[\left(\begin{array}{c}
-\frac{\alpha^{2}}{2}\left(L+L^{T}\right) \\
O_{N} \quad-\frac{\beta^{2}}{2}\left(L+L^{T}\right)+\alpha I_{N}
\end{array}\right) \otimes I_{n}\right] \tilde{y}(t) \\
& \leq \frac{\left(\rho_{1}+\rho_{2}+2 \rho_{3}-\alpha \lambda_{2}\left(L+L^{T}\right)\right) \alpha}{2} \sum_{i=1}^{N}\left\|\tilde{x}_{i}(t)\right\|^{2}+\left(\beta \rho_{3}+\alpha \rho_{4}\right) \sum_{i=1}^{N}\left\|\tilde{x}_{i}(t)\right\|\left\|\tilde{v}_{i}(t)\right\| \\
& +\frac{\left(\rho_{1}+\rho_{2}+2 \rho_{4}\right) \beta+2 \alpha-\beta^{2} \lambda_{2}\left(L+L^{T}\right)}{2} \sum_{i=1}^{N}\left\|\tilde{v}_{i}(t)\right\|^{2}+\frac{(\alpha+\beta) \rho_{1}}{2} \sum_{i=1}^{N}\left\|\tilde{x}_{i}(t-\tau)\right\|^{2} \\
& +\frac{(\alpha+\beta) \rho_{2}}{2} \sum_{i=1}^{N}\left\|\tilde{v}_{i}(t-\tau)\right\|^{2} \\
& =\frac{1}{2}\left(-\|\tilde{y}(t)\|^{T}\left(R_{1} \otimes I_{N}\right)\|\tilde{y}(t)\|+\|\tilde{y}(t-\tau)\|^{T}\left(S_{1} \otimes I_{N}\right)\|\tilde{y}(t-\tau)\|\right),
\end{aligned}
$$

where $R_{1}=\left(\begin{array}{cc}\left(\alpha \lambda_{2}\left(L+L^{T}\right)-\rho_{1}-\rho_{2}-2 \rho_{3}\right) \alpha & -\left(\beta \rho_{3}+\alpha \rho_{4}\right) \\ -\left(\beta \rho_{3}+\alpha \rho_{4}\right) & \beta^{2} \lambda_{2}\left(L+L^{T}\right)-\left(\rho_{1}+\rho_{2}+2 \rho_{4}\right) \beta-2 \alpha\end{array}\right), \quad S_{1}=\left(\begin{array}{cc}(\alpha+\beta) \rho_{1} & 0 \\ 0 & (\alpha+\beta) \rho_{2}\end{array}\right)$, $\|\tilde{x}(t)\|=\left(\left\|\tilde{x}_{1}(t)\right\|, \cdots,\left\|\tilde{x}_{N}(t)\right\|\right)^{T},\|\tilde{v}(t)\|=\left(\left\|\tilde{v}_{1}(t)\right\|, \cdots,\left\|\tilde{v}_{N}(t)\right\|\right)^{T},\|\tilde{y}(t)\|=\left(\|\tilde{x}(t)\|^{T},\|\tilde{v}(t)\|^{T}\right)^{T}, \quad\|\tilde{y}(t-\tau)\|=(\| \tilde{x}(t-$ $\left.\tau)\left\|^{T},\right\| \tilde{v}(t-\tau) \|^{T}\right)^{T}$. On the other hand, one has

$$
\begin{aligned}
V(t) & =\frac{1}{2} \tilde{y}^{T}(t)\left(P \otimes I_{n}\right) \tilde{y}(t) \\
& =\frac{\alpha \beta}{2} \tilde{x}^{T}(t)\left(\left(L+L^{T}\right) \otimes I_{n}\right) \tilde{x}(t)+\alpha \tilde{x}^{T}(t) \tilde{v}(t)+\frac{\beta}{2} \tilde{v}^{T}(t) \tilde{v}(t) \\
& \leq \frac{1}{2} \tilde{y}^{T}(t)\left(P_{1} \otimes I_{N n}\right) \tilde{y}(t),
\end{aligned}
$$

where $P_{1}=\left(\begin{array}{cc}\alpha \beta \lambda_{N}\left(L+L^{T}\right) & a \\ a & \beta\end{array}\right)$. Thus, according to Eq. (16) and the following facts:

$$
\begin{aligned}
V(t) & \leq \frac{1}{2} \lambda_{2}\left(P_{1}\right) \tilde{y}^{T}(t) \tilde{y}(t), \\
V(t-\tau) & \geq \frac{1}{2} \lambda_{1}(Q) \tilde{y}^{T}(t-\tau) \tilde{y}(t-\tau), \\
\|\tilde{y}(t)\|^{T} R_{1}\|\tilde{y}(t)\| & \geq \lambda_{1}\left(R_{1}\right) \tilde{y}^{T}(t) \tilde{y}(t), \\
\|\tilde{y}(t-\tau)\|^{T} S_{1}\|\tilde{y}(t-\tau)\| & \leq \lambda_{2}\left(S_{1}\right) \tilde{y}^{T}(t-\tau) \tilde{y}(t-\tau),
\end{aligned}
$$

one obtains

$$
\dot{V}(t) \leq-\gamma_{1} V(t)+\gamma_{2} V(t-\tau)
$$

where $\gamma_{1}=\frac{\lambda_{1}\left(R_{1}\right)}{\lambda_{2}\left(P_{1}\right)}, \quad \gamma_{2}=\frac{c_{0}}{\lambda_{1}(Q)}$, and $c_{0}=(\alpha+\beta) \max \left\{\rho_{1}, \rho_{2}\right\}$. 
For $k \omega+\delta<t<(k+1) \omega, \quad k \in \mathbb{N}$, taking the time derivative of $V(t)$ along the trajectories of (8) gives

$$
\begin{aligned}
\dot{V}(t)= & \tilde{y}^{T}(t)\left(P \otimes I_{n}\right)\left[F(x(t-\tau), v(t-\tau), x(t), v(t), t)+\left(B_{2} \otimes I_{n}\right) \tilde{y}(t)\right] \\
= & \alpha \tilde{x}^{T}\left[\left(I_{N}-\frac{1}{N} 1_{N \times N}\right) \otimes I_{n}\right] f(x(t-\tau), v(t-\tau), x(t), v(t), t)+\beta \tilde{v}^{T}\left[\left(I_{N}-\frac{1}{N} 1_{N \times N}\right) \otimes I_{n}\right] \\
& f(x(t-\tau), v(t-\tau), x(t), v(t), t)+\tilde{y}^{T}(t)\left[\left(B_{2} \otimes I_{n}\right)\right] \tilde{y}(t) .
\end{aligned}
$$

Similar to the previous analysis, one obtains

$$
\begin{aligned}
\dot{V}(t) & \leq \frac{\left(\rho_{1}+\rho_{2}+2 \rho_{3}\right) \alpha}{2} \sum_{i=1}^{N}\left\|\tilde{x}_{i}(t)\right\|^{2}+\frac{(\alpha+\beta) \rho_{1}}{2} \sum_{i=1}^{N}\left\|\tilde{x}_{i}(t-\tau)\right\|^{2}+\frac{\left(\rho_{1}+\rho_{2}+2 \rho_{4}\right) \beta}{2} \sum_{i=1}^{N}\left\|\tilde{v}_{i}(t)\right\|^{2} \\
& +\frac{(\alpha+\beta) \rho_{2}}{2} \sum_{i=1}^{N}\left\|\tilde{v}_{i}(t-\tau)\right\|^{2}+\left(\beta \rho_{3}+\alpha \rho_{4}\right) \sum_{i=1}^{N}\left\|\tilde{x}_{i}(t)\right\|\left\|\tilde{v}_{i}(t)\right\|+\tilde{y}^{T}(t)\left[\left(\begin{array}{cc}
O_{N} & I_{N} \\
O_{N} & O_{N}
\end{array}\right) \otimes I_{n}\right] \tilde{y}(t) \\
& =\frac{1}{2}\left(\|\tilde{y}(t)\|^{T}\left(R_{2} \otimes I_{N}\right)\|\tilde{y}(t)\|+\|\tilde{y}(t-\tau)\|^{T}\left(S_{2} \otimes I_{N}\right)\|\tilde{y}(t-\tau)\|\right),
\end{aligned}
$$

where $R_{2}=\left(\begin{array}{cc}\left(\rho_{1}+\rho_{2}+2 \rho_{3}\right) \alpha & \beta \rho_{3}+\alpha \rho_{4}+1 \\ \beta \rho_{3}+\alpha \rho_{4}+1 & \left(\rho_{1}+\rho_{2}+2 \rho_{4}\right) \beta\end{array}\right), \quad S_{2}=\left(\begin{array}{cc}(\alpha+\beta) \rho_{1} & 0 \\ 0 & (\alpha+\beta) \rho_{2}\end{array}\right)$. It then follows that

$$
\begin{aligned}
\dot{V}(t) & \leq \frac{\lambda_{2}\left(R_{2}\right)}{\lambda_{1}(Q)} V(t)+\frac{\lambda_{2}\left(S_{2}\right)}{\lambda_{1}(Q)} V(t-\tau) \\
& \leq \gamma_{3} V(t)+\gamma_{4} V(t-\tau)
\end{aligned}
$$

where $\gamma_{3}=\frac{c_{1}+c_{2}+\sqrt{\left(c_{1}-c_{2}\right)^{2}+c_{3}}}{\lambda_{1}(Q)}, \gamma_{4}=\frac{c_{0}}{\lambda_{1}(Q)}, c_{1}=\frac{\left(\rho_{1}+\rho_{2}+2 \rho_{3}\right) \alpha}{2}, c_{2}=\frac{\left(\rho_{1}+\rho_{2}+2 \rho_{4}\right) \beta}{2}, c_{3}=\left(\beta \rho_{3}+\alpha \rho_{4}+1\right)^{2}, c_{0}=(\alpha+$ $\beta) \max \left\{\rho_{1}, \rho_{2}\right\}$.

Based on the above analysis and according to Lemma 4, one obtains

$$
V(t) \leq|V(0)|_{\tau} e^{-r t}, \quad 0 \leq t \leq \delta,
$$

where $r$ is the unique positive solution of $-r=-\gamma_{1}+\gamma_{2} e^{r \tau},|V(0)|_{\tau}=\max _{-\tau \leq s \leq 0} V(s)$. For $\delta<t<\omega$, by using Lemma 5 , one obtains

$$
V(t) \leq|V(\delta)|_{\tau} e^{\left(\gamma_{3}+\gamma_{4}\right) t}
$$

Then, according to (22), one has

$$
|V(\delta)|_{\tau}=\max _{\delta-\tau \leq t \leq \delta} V(t) \leq|V(0)|_{\tau} e^{-r(\delta-\tau)}
$$

Combining (23) and (24) yields

$$
V(t) \leq|V(\delta)|_{\tau} e^{\left(\gamma_{3}+\gamma_{4}\right)(t-\delta)} \leq|V(0)|_{\tau} e^{-r(\delta-\tau)+\left(\gamma_{3}+\gamma_{4}\right)(t-\delta)}, \quad \delta<t<\omega .
$$

As $V(t)$ is a continuous function of $t$, one has

$$
V(\omega)=\lim _{t \rightarrow \omega^{-}} V(t) \leq|V(0)|_{\tau} e^{-r(\delta-\tau)+\left(\gamma_{3}+\gamma_{4}\right)(\omega-\delta)} .
$$

Then,

$$
\begin{aligned}
|V(\omega)|_{\tau} & =\max _{\omega-\tau \leq t \leq \omega} V(t) \\
& \leq|V(\delta)|_{\tau} e^{\left(\gamma_{3}+\gamma_{4}\right)(\omega-\delta)} \\
& \leq|V(0)|_{\tau} e^{-r(\delta-\tau)+\left(\gamma_{3}+\gamma_{4}\right)(\omega-\delta)}=|V(0)|_{\tau} e^{-\Delta}
\end{aligned}
$$


where $\Delta=r(\delta-\tau)-\left(\gamma_{3}+\gamma_{4}\right)(\omega-\delta)>0$. For any positive integer $k$, one has

$$
|V(k \omega)|_{\tau} \leq|V(0)|_{\tau} e^{-k \Delta}
$$

For arbitrary $t>0$, there exists a non-negative integer $k$, such that $k \omega<t \leq(k+1) \omega$. When $t \in(k \omega, k \omega+\delta]$, one obtains

$$
\begin{aligned}
V(t) & \leq|V(k \omega)|_{\tau} e^{-r(t-k \omega)} \\
& \leq|V(0)|_{\tau} e^{-k \Delta-r(t-k \omega)} \\
& \leq|V(0)|_{\tau} e^{-k \Delta} \\
& \leq|V(0)|_{\tau} e^{\Delta} e^{-\left(\frac{\Delta}{\omega}\right) t}
\end{aligned}
$$

When $t \in(k \omega+\delta,(k+1) \omega]$, one has

$$
\begin{aligned}
V(t) & \leq|V(k \omega+\delta)|_{\tau} e^{\left(\gamma_{3}+\gamma_{4}\right)(t-k \omega-\delta)} \\
& \leq|V(0)|_{\tau} e^{-k \Delta-r \delta} e^{\left(\gamma_{3}+\gamma_{4}\right)(\omega-\delta)} \\
& \leq|V(0)|_{\tau} e^{\left(\gamma_{3}+\gamma_{4}\right)(\omega-\delta)-r \delta+\Delta} e^{-\left(\frac{\Delta}{\omega}\right) t} \\
& =|V(0)|_{\tau} e^{-r \tau} e^{-\left(\frac{\Delta}{\omega}\right) t} .
\end{aligned}
$$

Combining (29)-(30) gives

$$
V(t) \leq K_{0} e^{-\left(\frac{\Delta}{\omega}\right) t}, \quad \text { for all } t>0
$$

where $K_{0}=e^{\Delta}|V(0)|_{\tau}$, which indicates that the states of agents exponentially converge to consensus. This completes the proof.

Corollary 1. Suppose that the communication topology $\mathcal{G}(A)$ is a strongly connected and balanced network, and Assumption 1 holds. Then, second-order consensus in system (5) is achieved if the following conditions hold:

(i) $\beta>\alpha$,

(ii) $\lambda_{2}\left(L+L^{T}\right)>\max \left\{\alpha^{-1}, \varrho_{1}, \varrho_{2}\right\}$,

(iii) $\delta>\frac{r \tau+\left(\gamma_{3}+\gamma_{4}\right) \omega}{r+\gamma_{3}+\gamma_{4}}$,

where $\varrho_{1}=\frac{\alpha\left(\rho_{1}+\rho_{2}+2 \rho_{3}\right)+\beta \rho_{3}+\alpha \rho_{4}}{\alpha^{2}}+\frac{\max \left\{\rho_{1}, \rho_{2}\right\}(\alpha+\beta)\left(\beta \lambda_{N}\left(L+L^{T}\right)+1\right)}{\alpha(\beta-\alpha)}, \quad \varrho_{2}=\frac{\beta\left(\rho_{1}+\rho_{2}+2 \rho_{4}\right)+2 \alpha}{\beta^{2}}+\frac{\max \left\{\rho_{1}, \rho_{2}\right\}(\alpha+\beta)\left[\alpha \beta \lambda_{N}\left(L+L^{T}\right)+\alpha\right]}{\beta^{2}(\beta-\alpha)}, r$ is the unique positive solution of $-r=-\gamma_{1}+\gamma_{2} e^{r \tau}, \gamma_{1}=\frac{\min \left\{\kappa_{1}, \kappa_{2}\right\}}{\alpha \beta \lambda_{N}\left(L+L^{T}\right)+\alpha}, \quad \gamma_{2}=\frac{(\alpha+\beta) \max \left\{\rho_{1}, \rho_{2}\right\}}{\beta-\alpha}, \quad \gamma_{3}=\frac{c_{1}+c_{2}+\sqrt{\left(c_{1}-c_{2}\right)^{2}+c_{3}}}{\beta-\alpha}$, $\gamma_{4}=\frac{(\alpha+\beta) \max \left\{\rho_{1}, \rho_{2}\right\}}{\beta-\alpha}, \quad \kappa_{1}=\beta^{2} \lambda_{2}\left(L+L^{T}\right)-\beta\left(\rho_{1}+\rho_{2}+2 \rho_{3}\right)-\left(\beta \rho_{3}+\alpha \rho_{4}\right), \kappa_{2}=\alpha^{2} \lambda_{2}\left(L+L^{T}\right)-\alpha\left(\rho_{1}+\rho_{2}+2 \rho_{4}\right)-2 \alpha$, $c_{1}=\frac{\left(\rho_{1}+\rho_{2}+2 \rho_{3}\right) \alpha}{2}, c_{2}=\frac{\left(\rho_{1}+\rho_{2}+2 \rho_{4}\right) \beta}{2}$, and $c_{3}=\left(\beta \rho_{3}+\alpha \rho_{4}+1\right)^{2}$.

Proof: Construct the same Lyapunov function candidate $V(t)$ as that in the proof of Theorem 1. By the Geršgorin disk theorem [43] and conditions (i) and (ii), the Corollary can be proved by following the proof of Theorem 1.

Remark 2. In Ref. [37], the concept of general algebraic connectivity $a(L)$ is introduced to describe the secondorder multi-agent system's ability to reach consensus. By Definition 6 in Ref. [37], one has $a(L)=\frac{\lambda_{2}\left(L+L^{T}\right)}{2}$ for a strongly connected and balanced $\mathcal{G}(A)$, where $L$ is the Laplacian matrix of the graph. Suppose that $\beta>\alpha$. Then, from the Corollary 1 , the second-order consensus can be achieved if the general algebraic connectivity $a(L)$ and the communication time duration $\delta$ are larger than their corresponding threshold values, respectively.

In practice, the communication topology among agents may not be fixed because of the restrictions of physical equipments or the signal interference. Therefore, it is more reasonable to assume that the communication topology 
is dynamically switching. Let $G=\left\{G\left(A_{1}\right), \cdots, G\left(A_{\pi}\right)\right\}$ be a set of possible topologies. For convenience, introduce a switching signal $\sigma:[0, \infty) \rightarrow \Pi$, where $\Pi=\{1, \cdots, \pi\}$. Denote by $L_{\sigma(t)}$ the Laplacian matrix of $G\left(A_{\sigma(t)}\right)$. Then, the following theorem and corollary can be obtained, for which the proofs are straight forward therefore omitted.

Theorem 2. Suppose that the communication topology $\mathcal{G}\left(A_{\sigma(t)}\right)$ is kept strongly connected and balanced throughout the process and, moreover, Assumption 1 holds. Then, second-order consensus in system (5) is achieved if the following conditions hold:

(i) $\lambda_{2}\left(L_{i}+L_{i}^{T}\right)>\frac{\alpha^{2}}{\beta}$,

(ii) $\lambda_{1}\left(R_{1}^{i}\right)>\frac{c_{0} \lambda_{2}\left(P_{1}^{i}\right)}{\lambda_{1}\left(Q^{i}\right)}$,

(iii) $\delta>\frac{r^{i} \tau+\left(\gamma_{3}^{i}+\gamma_{4}^{i}\right) \omega}{r^{i}+\gamma_{3}^{i}+\gamma_{4}^{i}}$,

where $R_{1}^{i}=\left(\begin{array}{cc}\left(\alpha \lambda_{2}\left(L_{i}+L_{i}^{T}\right)-\rho_{1}-\rho_{2}-2 \rho_{3}\right) \alpha & -\left(\beta \rho_{3}+\alpha \rho_{4}\right) \\ -\left(\beta \rho_{3}+\alpha \rho_{4}\right) & \beta^{2} \lambda_{2}\left(L_{i}+L_{i}^{T}\right)-\left(\rho_{1}+\rho_{2}+2 \rho_{4}\right) \beta-2 \alpha\end{array}\right), P_{1}^{i}=\left(\begin{array}{cc}\alpha \beta \lambda_{\max }\left(L_{i}+L_{i}^{T}\right) & \alpha \\ \alpha & \beta\end{array}\right)$,

$Q^{i}=\left(\begin{array}{cc}\alpha \beta \lambda_{2}\left(L_{i}+L_{i}^{T}\right) & \alpha \\ \alpha & \beta\end{array}\right), c_{0}=(\alpha+\beta) \max \left\{\rho_{1}, \rho_{2}\right\}$, and $r^{i}$ is the unique positive solution of $-r^{i}=-\gamma_{1}^{i}+\gamma_{2}^{i} e^{r^{i} \tau}$,

$\gamma_{1}^{i}=\frac{\lambda_{1}\left(R_{1}^{i}\right)}{\lambda_{2}\left(P_{1}^{i}\right)}, \gamma_{2}=\frac{c_{0}}{\lambda_{1}\left(Q^{i}\right)}, \gamma_{3}^{i}=\frac{c_{1}+c_{2}+\sqrt{\left(c_{1}-c_{2}\right)^{2}+c_{3}}}{\lambda_{1}\left(Q^{i}\right)}, \gamma_{4}^{i}=\frac{c_{0}}{\lambda_{1}\left(Q^{i}\right)}, \quad c_{1}=\frac{\left(\rho_{1}+\rho_{2}+2 \rho_{3}\right) \alpha}{2}, c_{2}=\frac{\left(\rho_{1}+\rho_{2}+2 \rho_{4}\right) \beta}{2}, c_{3}=\left(\beta \rho_{3}+\alpha \rho_{4}+\right.$ $1)^{2}, i \in \Pi$.

Corollary 2. Suppose that the communication topology $\mathcal{G}\left(A_{\sigma(t)}\right)$ is kept strongly connected and balanced throughout the process and, moreover, Assumption 1 holds. Then, second-order consensus in system (5) is achieved if the following conditions hold:

(i) $\beta>\alpha$,

(ii) $\min _{i \in \Pi} \lambda_{2}\left(L_{i}+L_{i}^{T}\right)>\max \left\{\alpha^{-1}, \varrho_{0}\right\}$,

(iii) $\delta>\frac{\bar{r} \tau+\left(\bar{\gamma}_{3}+\bar{\gamma}_{4}\right) \omega}{\bar{r}+\bar{\gamma}_{3}+\bar{\gamma}_{4}}$,

where $\varrho_{0}=\max _{i \in \Pi}\left\{\varrho_{1}^{i}, \varrho_{2}^{i}\right\}, \quad \varrho_{1}^{i}=\frac{\alpha\left(\rho_{1}+\rho_{2}+2 \rho_{3}\right)+\beta \rho_{3}+\alpha \rho_{4}}{\alpha^{2}}+\frac{\rho_{0}(\alpha+\beta)\left(\beta \lambda_{N}\left(L_{i}+L_{i}^{T}\right)+1\right)}{\alpha(\beta-\alpha)}, \quad \varrho_{2}^{i}=\frac{\beta\left(\rho_{1}+\rho_{2}+2 \rho_{4}\right)+2 \alpha}{\beta^{2}}+\frac{\rho_{0}(\alpha+\beta)\left[\alpha \beta \lambda_{N}\left(L_{i}+L_{i}^{T}\right)+\alpha\right]}{\beta^{2}(\beta-\alpha)}$, $\bar{r}$ is the unique positive solution of $-\bar{r}=-\bar{\gamma}_{1}+\bar{\gamma}_{2} e^{\bar{r} \tau}, \bar{\gamma}_{1}=\frac{\kappa_{\min }}{\alpha \beta \lambda_{N}\left(L+L^{T}\right)+\alpha}, \quad \bar{\gamma}_{2}=\frac{(\alpha+\beta) \max \left\{\rho_{1}, \rho_{2}\right\}}{\beta-\alpha}, \bar{\gamma}_{3}=\frac{c_{1}+c_{2}+\sqrt{\left(c_{1}-c_{2}\right)^{2}+c_{3}}}{\beta-\alpha}$, $\bar{\gamma}_{4}=\frac{(\alpha+\beta) \max \left\{\rho_{1}, \rho_{2}\right\}}{\beta-\alpha}, \kappa_{\min }=\min _{i \in \Pi}\left\{\kappa_{1}^{i}, \kappa_{2}^{i}\right\}, \kappa_{1}^{i}=\beta^{2} \lambda_{2}\left(L_{i}+L_{i}^{T}\right)-\beta\left(\rho_{1}+\rho_{2}+2 \rho_{3}\right)-\left(\beta \rho_{3}+\alpha \rho_{4}\right), \kappa_{2}^{i}=\alpha^{2} \lambda_{2}\left(L_{i}+L_{i}^{T}\right)-$ $\alpha\left(\rho_{1}+\rho_{2}+2 \rho_{4}\right)-2 \alpha, c_{1}=\frac{\left(\rho_{1}+\rho_{2}+2 \rho_{3}\right) \alpha}{2}, c_{2}=\frac{\left(\rho_{1}+\rho_{2}+2 \rho_{4}\right) \beta}{2}, c_{3}=\left(\beta \rho_{3}+\alpha \rho_{4}+1\right)^{2}, i \in \Pi$.

\section{A Simulation Example}

In this section, a simulation example is provided to verify the theoretical analysis.

Consider the second-order consensus protocol with time-delayed nonlinear velocities in system (5), where the communication topology is shown in Fig. 1 with weighting on the edges. The time-delayed nonlinear function $f$ is described by time-delayed Chua's circuit [44]:

$$
f\left(x_{i}(t-\tau), v_{i}(t-\tau), x_{i}(t), v_{i}(t), t\right)=\left(\begin{array}{l}
\mu\left(-v_{i 1}+v_{i 2}-l\left(v_{i 1}\right)\right) \\
v_{i 1}-v_{i 2}+v_{i 3} \\
-\varsigma v_{i 2}-\epsilon \sin \left(\sigma v_{i 1}(t-\tau)\right)
\end{array}\right), \quad i=1, \cdots, 4,
$$

where $l\left(v_{i 1}\right)=b v_{i 1}+0.5(a-b)\left(\left|v_{i 1}+1\right|-\left|v_{i 1}-1\right|\right), x_{i}=\left[x_{i 1}, x_{i 2}, x_{i 3}\right]^{T}, v_{i}=\left[v_{i 1}, v_{i 2}, v_{i 3}\right]^{T}$. The isolated system (32) is chaotic when $\mu=10, \varsigma=18, \epsilon=0.02, \sigma=0.02, \tau=0.01, a=-4 / 3$ and $b=-3 / 4$, as shown in Fig. 1 with initial conditions $v_{i}(t)=[0.016,0.018,-0.015]^{T}, t \in[-\tau, 0]$. In view of Assumption 1 , one obtains $\rho_{1}=0, \rho_{2}=0.0004, \rho_{3}=0, \rho_{4}=4.3871$. Let $\alpha=11.5, \beta=12, \delta=0.485$, and $\omega=0.5$. From Fig. 2, 
it is easy to see that the communication topology $\mathcal{G}(\mathcal{A})$ is strongly connected and balanced. By Theorem 1 , one has $\lambda_{2}\left(L+L^{T}\right)=12>\frac{\alpha^{2}}{\beta}=11.0208, \lambda_{1}\left(R_{1}\right)=1555.7>\frac{c_{0} \lambda_{2}\left(P_{1}\right)}{\lambda_{1}(Q)}=3.0473$, and $\delta=0.485>\frac{r \tau+\left(\gamma_{3}+\gamma_{4}\right) \omega}{r+\gamma_{3}+\gamma_{4}}=$ 0.4845 . Therefore, second-order consensus can be achieved in multi-agent system (5). The position and velocity states of all agents are shown in Fig. 2, with initial conditions $x_{1}(t)=[0.25,-0.13,0.04]^{T}, x_{2}(t)=[2,1.5,2.5]^{T}$, $x_{3}(t)=[-1,-1.5,-2.5]^{T}, x_{4}(t)=[-2,-0.8,0.3]^{T}, v_{1}(t)=[3.016,2.018,0.085]^{T}, v_{2}(t)=[2.016,3.018,1.085]^{T}$, $v_{3}(t)=[-1.085,-1.282,1.285]^{T}, v_{4}(t)=[-2.085,-0.582,-0.015]^{T}$, for $t \in[-\tau, 0]$. Simulation results shown in Figs. (3) and (4) verify the theoretical analysis very well.

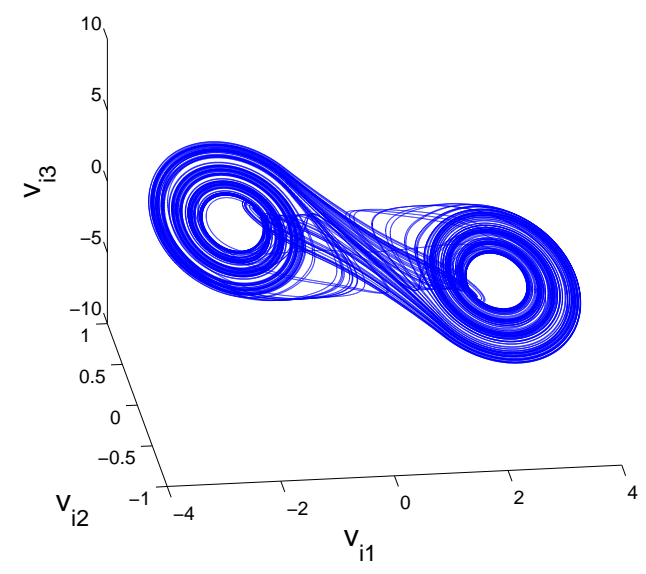

Figure 1: Chaotic trajectory of the model (32).

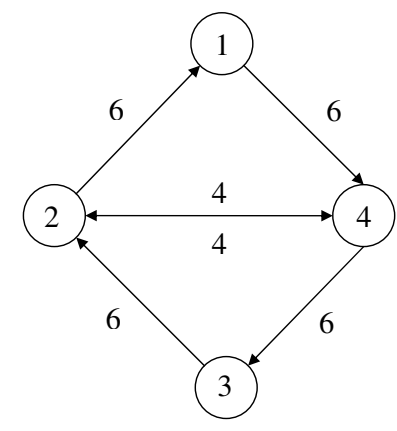

Figure 2: Communication topology $\mathcal{G}(\mathcal{A})$.

\section{Conclusions}

In this paper, a novel second-order intermittent consensus protocol for multi-agent systems with time-delayed nonlinear dynamics and switching communication topologies has been introduced and studied. It has been shown that second-order consensus can be reached if the communication time duration and the general algebraic connectivity are larger than their corresponding thresholds, respectively. Future work will be focused on the consensus behaviors 


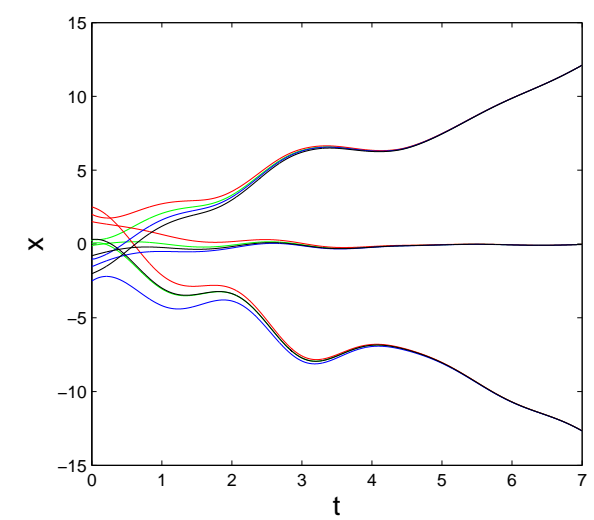

Figure 3: Consensus of state trajectories of multiple agents.

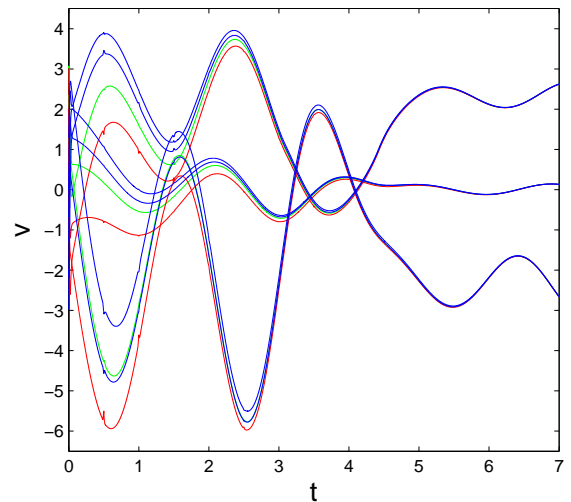

Figure 4: Consensus of velocity trajectories of multiple agents.

of more complicated and practical models, such as second-order multi-agent systems with nonlinear dynamics and transmission delays, higher-order multi-agent systems with nonlinear dynamics, and so on.

\section{References}

[1] R.W. Beard, T. W. McLain, M. A. Goodrich, and E. P. Anderson. "Coordinated target assignment and intercept for unmanned air vehicles," IEEE Trans. Robotics Automat., vol. 18, no. 6, pp. 911-922 (2002).

[2] A. Fax and R. M. Murray, "Information flow and coorperative control of vehicle formations," IEEE Trans. Automat. Contr., vol. 49, no. 9, pp. 1465-1476 (2004).

[3] W. Ren, R. W. Beard, and E. M. Atkins, ’Information consensus in multivehicle cooperative control,” IEEE Control Syst. Mag., Vol. 27, no. 2, pp. 71-82 (2007).

[4] J. Cortes and F. Bullo, "Coordination and geometric optimization via distributed dynamical systems," SIAM J. Control Optim., vol. 44, no. 5, pp. 1543-1574 (2003).

[5] T. Vicsek, A. Cziroók, E. Ben-Jacob, O. Cohen, and I. Shochet, "Novel type of phase transition in a system of self-deriven particles," Phys. Rev. Lett., vol. 75, no. 6, pp. 1226-1229 (1995).

[6] J. Toner and Y. Tu, "Flocks, herds, and school: a quantitative theory of flocking," Phys. Rev. E, vol. 58, no. 4, pp. 4828-4858 (1998).

[7] C. M. Topaz and A. L. Bertozzi, "Swarming patterns in a two-dimensional kinematic model for biological groups," SIAM J. Appl. Math., vol.65, pp. 152-174 (2005).

[8] A. Jadbabaie, J. Lin, and A. S. Morse, "Coordination of groups of moboile autonomous agents using nearest neighbour rules, IEEE Trans. Automat. Contr., vol. 48, vol. 9, pp. 988-1001 (2003). 
[9] R. Olfati-Saber, R. M. Murray, "Consensus problems in networks of agents with switching topology and time-delays," IEEE Trans. Automat. Contr., vol. 49, vol. 9, pp. 1520-1533 (2004).

[10] W. Ren and R. W. Beard, "Consensus seeking in multiagent systems under dynamically changing interaction topologies," IEEE Trans. Automat. Contr., vol. 50, vol. 5, pp. 655-661 (2005).

[11] Y. Hatano and M. Mesbahi, ”Agreement over random networks," IEEE Trans. Automat. Contr., vol. 50, no. 11, pp. 1867-1872 (2005).

[12] M. Porfiri and D. J. Stilwell, "Consensus seeking over random weighted Directed Graphs", IEEE Trans. Automat. Contr., vol. 52, no. 9, pp. 1767-1773 (2007).

[13] F. Xiao and L. Wang, "Asynchronous consensus in continuous-time multi-agent systems with switching topology and time-varying delays," IEEE Trans. Automat. Contr., vol. 53, no. 8, pp. 1804-1816 (2008).

[14] L. Fang and P. J. Antsaklis, "Asynchronous consensus protocols using nonlinear paracontractions theory," IEEE Trans. Automat. Contr., vol. 53, no. 10, pp. 2351-2355 (2008).

[15] M. Cao, A.S. Morse, and B.D.O. Anderson, "Reaching a consensus in a dynamically changing environment: a graphical approach," SIAM J. Contr. Optim., vol. 47, no. 2, pp. 575-600 (2008).

[16] F. Xiao and L. Wang, "Consensus problems for high-dimensional multi-agents systems", IET Control Theory Appl., vol. 1, no. 3, pp. 830-837 (2007).

[17] Q. Hui, and W. M. Haddad, ”Distributed nonlinear control algorithms for networks consensus”, Automatica, vol. 44, no. 9, pp. 2375-2381 (2008).

[18] U. Münz, A. Papachristodoulou, F. Allgöwer, "Consensus reaching in multi-agent packet-switched networks with non-linear coupling," Int. J. Contr., vol. 82, no. 5, pp. 953-969 (2009).

[19] T. Li and J. F. Zhang, "Mean square average consensus under measurement noises and fixed topologies: necessary and sufficient conditions," Automatica, vol. 45, no.8, pp. 1929-1936 (2009).

[20] T. Li and J. F. Zhang, "Consensus conditions of multi-agent systems with time-varying topologies and stochastic communication noises," IEEE Trans. Automat. Contr., in press.

[21] G. Xie, and L. Wang, "Consensus control for a class of networks of dynamic agents: fixed topology," Proc. of the IEEE Conference on Decision and Control, pp. 96-101 (2005).

[22] W. Ren, and E. Atkins, "Second-order consensus protocols in multiple vehicle systems with local interactions," Proc. of AIAA Guidance, Navigation, and Control, AIAA-2005-6238 (2005).

[23] W. Ren, and E. Atkins, "Distributed multi-vehicle coordinated control via local information exchange," Int. J. Robust and Nonlinear Control, vol. 17, no. 10-11, pp. 1002-1033 (2007)

[24] W. Ren, and E. Atkins, "On consensus algorithms for double-integratos dynamics," IEEE Trans. Automat. Contr., vol. 58, no. 6, pp. 1503-1509 (2008).

[25] Y. Hong, J. Hu, and L. Gao, "Tracking control for multi-agent consensus with an active leader and varibale topology" Automatica, vol. 42, no. 7, pp. 1177-1182 (2006).

[26] Y. Hong, G. Chen, and L. Bushnell, ’Distributed observers design for leader-following control of multi-agent networks," Automatica, vol. 44, no. 3, pp. 846-850 (2008).

[27] P. Wieland, J.-S., kim, H. Scheu, and F. Allgöwer, "On consensus in multi-agent systems with linear high-order agents", Proc. of the 17th world congress, pp. 1541-1546 (2008).

[28] H. Liu, G. Xie, and L. Wang, "Necessary and sufficient conditions for solving consensus problems of double-integrator dynamics via sampled control", Int. J. Robust and Nonlinear Control, DOI: 10.1002/rnc.1543.

[29] W. Yu, G. Chen, and M. Cao, "Some necessary and sufficient conditions for second-order consensus in multi-agent dynamical systems", Automatica, in press.

[30] W. Ren, "Synchronization of coupled harmonic oscillators with local interaction", Automatica, vol. 44, no. 16, pp. 3195-3200 (2008).

[31] X. Wang, and G. Chen, "Synchronization in scale-free dynamical networks: robustness and fragility," IEEE Trans. Circuits Syst. I, vol. 49, no. 1, pp. 54-62 (2002).

[32] X. Wang, and G. Chen, ”Synchronization in small-world dynamical networks," Int. J. Bifur. Chaos, vol. 12, no. 1, pp. 187-192 (2002).

[33] J. Lü, and G. Chen, "A time-varying complex dynamical network models and its controlled syncyhronization criteria," IEEE Trans. Auto. Contr., vol. 50, no. 6, pp. 841-846 (2005).

[34] J. Zhou, J. Lu, and J. Lü, "Adaptive synchronization of an uncertainty complex dynamical netwok," IEEE Trans. Auto. Contr., vol. 51, no. 4, pp. 652-656 (2006).

[35] J. Cao, G. Chen, and P. Li, "Global synchronization in an array of delayed neural networks with hybrid coupling," IEEE Trans. Systems, Man, and Cerbernetics-Part B, vol. 38, no. 2, pp. 488-498 (2008).

[36] W. Lu, and T. Chen, "New approach to synchronization analysis of linearly coupled ordinary differential systems", Physica D, vol. 213, no. 
2, pp. 214-230 (2006).

[37] W. Yu, G. Chen, M. Cao, and J. Kurths, "Second-order cosnensus for multi-agent systems with directed topologies and nonlinear dynamics", IEEE Trans. Systems, Man, and Cybernetics-Part B, in press.

[38] V. Gazi, and K. M. Passino, "Stability analysis of swarms", IEEE Trans. Automat. Contr, vol. 48, no. 4, pp. $692-697$ (2003).

[39] R. Olfati-Saber,"Flocking for multi-agent dynamic systems: algorithms and theory", IEEE Trans. Automat. Contr., vol. 51, no. 3, pp. 401-420 (2006).

[40] S. Boyd, L.E. Ghaoui, E. Ferion, and V. Balakrishnan, Linear Matrix Inequalities in System and Control Theory, Philadelphia, PA: SIAM, (1994).

[41] H. Fujisaka, and T. Yamada, "Stability theory of synchronized motion in coupled-oscillator systems", Prog. Theor. Phys., vol. 69, no. 1, pp. 32-47 (1983).

[42] T. Huang, C. Li, W. Yu, and G. Chen, "Synchronization of delayed chaotic systems with parameter mismatches by using intermittent linear state feedback", Nonlinearity, vol. 22, no. 3, pp. 569-584 (2009).

[43] R. A. Horn and C. R. Johnson, Matrix Analysis, Cambridge, U.K.: Cambridge Univ. Press, (1985).

[44] X. Wang, G. Zhong, K. Tang, K. Man, and Z. Liu, "Anticontrol of chaos in continuous-time systems via time-delay feedback," Chaos, vol. 10, no. 4, pp. 771-779 (2000). 